\title{
Afterglow Plasma Diagnostics with a Microwave Sampling Radiometer
}

\author{
R. L. Stenzel and R. W. Gould \\ California Institute of Technology, Pasadena, California 91109
}

(Received 6 June 1969)

\begin{abstract}
A simple waveguide arrangement has been developed for the study of microwave absorption and emission from a magnetized afterglow plasma column. The time and frequency resolved measurements are performed by a sampling radiometer. A comparison and null technique permits the direct measurement of the electron temperature. Continuous plots of the temperature vs frequency, magnetic field, and afterglow time are made possible by means of a servoloop. The width of the emission or absorption spectrum in the range of upper hybrid frequencies is used to derive the electron density which, together with the temperature measurement, allows a more complete analysis of the plasma decay.
\end{abstract}

\section{INTRODUCTION}

M ICROWAVE radiometers have been used for a number of years to measure noise emission in transient processes. ${ }^{1}$ Their basic design goes back to Dicke's switched radiometer ${ }^{2}$ which compares the unknown noise signal with a calibrated reference noise signal. The measurement is made by adjusting the reference signal to equal the unknown signal to yield zero radiometer output. This null technique has been retained in a sampling radiometer, while the simple alternate switching from test to reference source has been modified to short duration sampling of both sources. The sampling is performed by gating one of the radiometer's amplifier stages, typically an i.f. stage.

Most of the time gated radiometers still use a ferrite switch at the input since the test channel and the reference channel are physically separated. However, in the present case the transient noise signal and a gated reference signal have been separated in time so that by using a suitable waveguide setup both signals are measured in a single channel, eliminating the need for a ferrite switch and thereby simplifying the instrumentation and improving the receiver's noise figure by the insertion loss of the switch.

The noise sampling and comparison technique was developed mainly for the measurement of the radiation temperature in a partially reflecting plasma. However, with minor modifications in the waveguide system and the reference signals, the method proved to be applicable for a number of other measurements. These include the emitted noise power, the power absorption coefficient, the power reflection coefficient, and, using standard slotted line techniques, the complex scattering parameters of the plasma column in the waveguide.

The plasma column passes through the waveguide in such a way that the incident electromagnetic waves

${ }^{1}$ D. Formato and A. Gilardini, Proc. Fourth Int. Conf. Ionization Phenomena Gases, Uppsala, Sweden Vol. 1, 1 A99 (1960); J.C. Ingraham and J. J. McCarthy, RLE Quart. Progr. Rep. No. 64, MIT 1962, p. 76; B. L. Wright, RLE Quart. Progr. Rep. No 80, MIT 1966 p. 99; J. H. Noon, P. R. Blaszuk, and E. H. Holt, J. Appl. Phys. 39, 9 (1968)

${ }_{2}^{2}$ R. H. Dicke, Rev. Sci. Instrum. 17, 268 (1946). strongly excite plasma resonances. In the frequency range of the upper hybrid resonances the plasma column has a strong absorptivity and emissivity which are crucial for the electron temperature measurement. The electron density is derived from the upper hybrid resonance condition. Thus, emission and absorption measurements are used for plasma diagnostics.

The main emphasis in this paper is on the experimental setup and the measurement technique. The basic properties of the plasma resonance are mentioned to show how the measurements are used for diagnostic work. A typical result is shown and further applications are indicated in the conclusion.

\section{SAMPLING RADIOMETER}

For completeness we briefly describe the radiometer. The block diagram is shown in Fig. 1. The input stage consists of a superheterodyne receiver. Both the signal and the image sideband of the receiver are used. This eliminates the need for a tunable microwave filter and improves the noise figure by at least $3 \mathrm{~dB}$ compared to a single sideband receiver. The center frequency of the receiver is easily tuned by changing the local oscillator frequency. The ambiguity in frequency response has been minimized by choosing a low intermediate frequency with a wide bandwidth. (In our case the $3 \mathrm{~dB}$ i.f. frequencies are 3.5 and $10 \mathrm{MHz}$.) The resultant microwave receiver response approximates a rectangular bandpass of $20 \mathrm{MHz}$ width

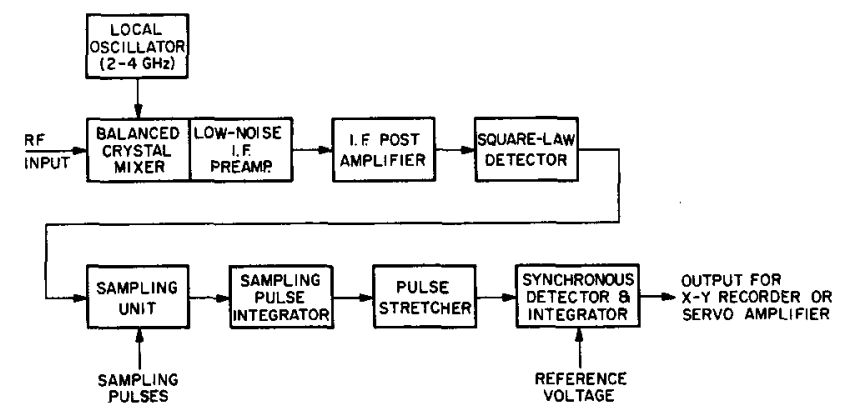

FIG. 1. Block diagram of the time gated radiometer. 
except for a notch around the center frequency. The frequency spread is less than $1 \%$ at S-band frequencies which is sufficient for the present noise measurements.

The amplified i.f. noise is applied to a crystal detector operating in the square law regime. An RC filter eliminates the high frequency components at the detector output. The low level video signal is amplified to a few volts and applied to a sampling unit, consisting of a high series resistor followed by a transistor shunt switch. The duty cycle of the noise samples (typically $10^{-3}$ ) is increased by pulse stretching. This operation is performed in two steps. An RC filter forms the average value of the sampled, detected noise over the sampling width. A pulse stretcher holds this value for a time long compared to the sampling width, but shorter than the sampling repetition time.

The stretched noise samples are applied to a synchronous detector (such as a PAR lock-in amplifier) which selects a desired frequency component from the sampling pulse spectrum and averages it over a time $\tau$. In Sec. IV we show that the output becomes proportional to the noise power difference between the unknown signal and a reference signal.

The radiometer sensitivity is limited by output fluctuations. By definition, the smallest detectable noise power difference produces an average output signal equal to the rms output fluctuations. When expressed in blackbody noise temperatures $(P=k T B)$ the sensitivity was found to be

$$
\Delta T=-\frac{\pi}{4}\left[\frac{\left(T_{\mathrm{s}}+T_{r}\right)^{2}+\left(T_{\mathrm{ref}}+T_{r}\right)^{2}}{B \tau \Delta t_{\mathrm{s}} / t_{\mathrm{s}}}\right]^{\prime},
$$

where the terms and some typical values are shown in Table I.

\section{WAVEGUIDE SETUP}

The measurements are concerned with the extraordinary waves in an axially magnetized afterglow plasma column. The waveguide arrangement serves to excite these waves and permits observation of reflected and emitted waves.

The plasma column passes perpendicularly through the narrow sides of a rectangular S-band waveguide (Fig. 2). In the unperturbed fundamental waveguide mode $\left(\mathrm{TE}_{10}\right)$ the incident electric field, the propagation vector, and the static magnetic field parallel to the column axis are all

TABLE I. Typical data and performance of the sampling radiometer. Observed and calculated sensitivity are in fair agreement.

\begin{tabular}{lc}
\hline \hline Predetection bandwidth & $B=7.5 \mathrm{MHz}$ \\
Sampling pulse width & $\Delta t_{s}=20 \mu \mathrm{sec}$ \\
Sampling repetition time & $t_{s}=20 \mathrm{msec}$ \\
Integration time & $\tau=1 \mathrm{sec}$ \\
Receiver noise temperature & $T_{r}=530 \mathrm{~K}$ \\
Signal noise temperature & $T_{s}=500 \mathrm{~K}$ \\
Reference noise temperature & $T_{r \mathrm{rf}}=290 \mathrm{~K}$ \\
Radiometer sensitivity & $\Delta T=12 \mathrm{~K},\left(\Delta P=2.5 \times 10^{-15} \mathrm{~W}\right)$ \\
\hline
\end{tabular}

perpendicular to one another, leading to a strong coupling of the waveguide fields to the extraordinary waves in the plasma.

Considering the power flow in the waveguide, the discontinuity created by the traversing plasma column can be described by an absorption coefficient $A^{\prime}$, a reflection coefficient $R^{\prime}$, and a transmission coefficient $T^{\prime}$. Two of these coefficients are independent since power conservation requires $A^{\prime}+R^{\prime}+T^{\prime}=1$. Two measurements would be necessary to determine the coefficients.

The transmitted wave can be made to interact again with the plasma column by terminating the waveguide in a perfect short. The incident waves are then reflected and absorbed, but not transmitted, and the resultant waveguide termination (short and discontinuity) is char-

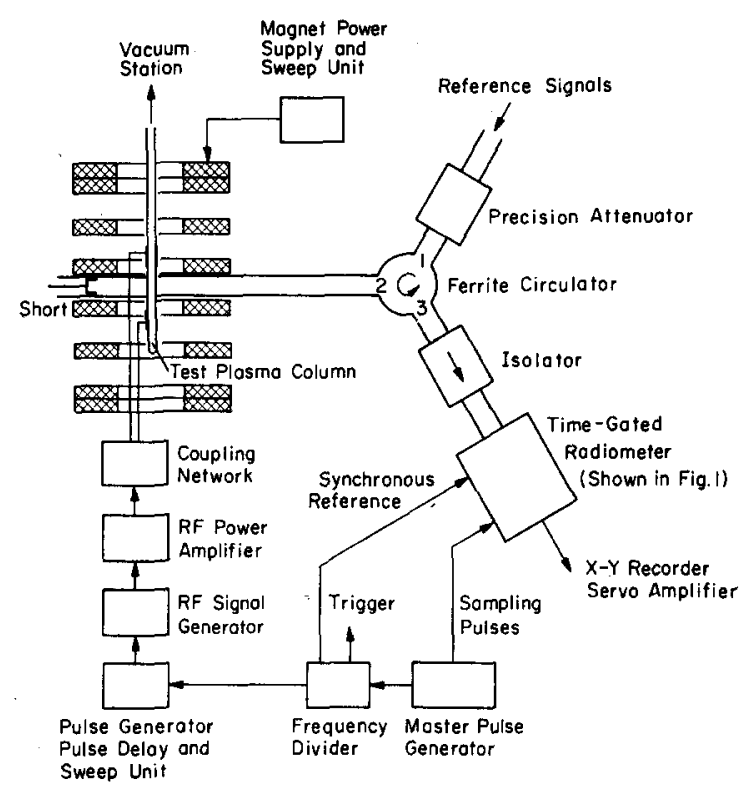

FIG. 2. Block diagram of the main elements for sampling and comparison measurements on a magnetized afterglow plasma column.

acterized by only two coefficients $A$ and $R$. Thus, the interactions are enhanced and a single measurement is sufficient to describe them $(A+R=1) .^{3}$ The absorption coefficient can be maximized by adjusting the position of the waveguide short.

The transient nature of the pulsed afterglow plasma allows the measurement of the unknown and the reference signals in a single waveguide on a time sharing basis. At time intervals when the test plasma has completely decayed a gated reference signal is incident which is totally reflected by the waveguide short.

\footnotetext{
${ }^{3}$ The idea of using a shorted waveguide for a strongly reflecting plasma was used earlier by J. C. Ingraham (Ph.D. thesis, MIT Dept. of Physics, 1963, unpublished). The present setup is simpler and arranges the plasma column in a new way so as to excite the upper hybrid resonances.
} 
The reflected signal is separated from the incident signal by means of a waveguide three-port ferrite circulator. The incident signal is introduced into port 1 . The power level can be accurately controlled by a precision attenuator. The reflected power leaves at port 3 , passes through two waveguide isolators, and enters the radiometer. A high isolation between the radiometer's local oscillator and the plasma column is required in order to avoid electron heating in the resonance range.

\section{PLASMA GENERATION AND ELECTRONIC EQUIPMENT}

The plasma is produced by gas breakdown with $\mathrm{rf}$ electric fields. The rf field is pulsed and the measurements are performed during the decay of the plasma (afterglow). The frequency $(20 \mathrm{MHz})$ lies in the range of the electronneutral collision frequency resulting in low breakdown field strengths. The rf signal is produced by a pulsed low power signal generator. A driver and rf power amplifier supply $500 \mathrm{~W}$ breakdown pulses of typically $100 \mu \mathrm{sec}$ width. The high of power results in a stable, repeatable plasma production from pulse to pulse. The rf power is coupled via a transmission line to a resonant circuit. Two cylindrical electrodes which fit tightly on the quartz tube containing the gas are connected to symmetric taps on the coil of the ungrounded resonant circuit. Complete shielding of all rf elements prevents interference with other sensitive instruments.

The plasma is contained in a cylindrical quartz tube ( $2 \mathrm{~cm}$ i.d., $50 \mathrm{~cm}$ discharge length) connected to a standard high vacuum station. Rare gases in the pressure range of 5 to $100 \mathrm{~m}$ Torr have been investigated. The plasma column is located axial to a uniform static magnetic field produced by an eight-coil air-core solenoid. The magnetic field can be varied from $B=0$ to $B=2 \mathrm{kG}$ by adjusting the regulated magnet current.

The sampling technique requires synchronization of all pulsed signals. A master pulse generator supplies pulses at a rate of $50 \mathrm{~Hz}$. These pulses determine width and repetition time for the sampling unit of the radiometer. A frequency divider (flipflop) generates $25 \mathrm{~Hz}$ square waves which are used as reference signals for the synchronous detector. They also trigger a pulse generator required to gate the rf breakdown signal. A variable pulse delay determines the time in the afterglow at which the measurement sample is taken. The delay is swept automatically so that continuous plots vs afterglow time can be taken.

Reference signals can be gated at a rate of $25 \mathrm{~Hz}$. A solid state noise source $\left(290 \mathrm{~K}<T_{\text {ref }}<100000 \mathrm{~K}\right)$ or a microwave signal generator is used as a reference source.

For the radiation temperature measurement the radiometer operates as a null detector. In order to make continuous plots of the temperature, a servoloop maintains

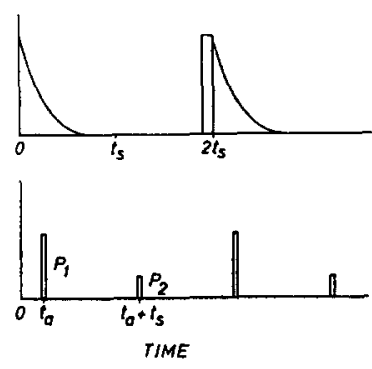

FIG. 3. Typical time sequence for afterglow noise sampling. The upper part represents the plasma noise emission during breakdown pulse and afterglow. The lower picture shows the radiometer's noise power samples. For each breakdown period $\left(0<t<2 t_{s}\right)$ a signal sample $\left(\mathrm{P}_{1}\right)$ and a reference sample $\left(\mathrm{P}_{2}\right)$ are taken.

zero radiometer output while other parameters are varied. The radiometer output voltage is amplified in a dc amplifier and controls the direction of rotation of a servomotor. The motor adjusts the precision attenuator which determines the reference noise power so as to yield zero radiometer output. A voltage, proportional to the attenuator position and calibrated in temperature units, is supplied for the vertical axis of an $\mathrm{X}-\mathrm{Y}$ recorder.

\section{MEASUREMENT TECHNIQUES}

A typical time sequence for the sampling and comparison method of afterglow measurements is shown in Fig. 3. The repetition time for the pulsed plasma generation is chosen sufficiently long so that the afterglow lies within the first half-period. The radiometer takes two samples per breakdown period. The first or signal sample $P_{\mathbf{1}}$ is taken at the desired afterglow time $t_{a}$ where $t_{a}=0$ corresponds to the end of a breakdown pulse. The second or reference sample is taken at $t_{a}+t_{s}$ where $t_{s}$ is the sampling repetition time. It contains only contributions from reference sources. ${ }^{4}$

Since the synchronous detector is supplied with a reference frequency $1 / 2 t_{s}$, the radiometer output is proportional to the fundamental Fourier component of the sample spectrum, i.e., proportional to the sample difference $\Delta P=P_{1}-P_{2}$. The contribution of the uncorrelated receiver noise which adds to each sample is canceled by taking the difference.

The noise emission from the plasma is measured easily. When the reference signal is zero the radiometer output is directly proportional to the noise power emitted by the plasma column in the waveguide which is given by ${ }^{5}$

$$
\Delta P=A k T_{\mathrm{rad}} B,
$$

where $A$ is the power absorption coefficient, $k$ the Boltzmann constant, $T_{\text {rad }}$ the electron radiation temperature,

\footnotetext{
${ }^{4}$ A similar technique has also been used for optical absorption measurements by A. V. Phelps and J. L. Pack, Rev. Sci. Instrum. 26, 45 (1955).

${ }^{5} \mathrm{G}$. Bekefi, Radiation Processes in Plasmas (John Wiley \& Sons, Inc., New York, 1966).
} 
and $B$ the bandwidth of the observed noise spectrum. For a blackbody the absorption coefficient is one. The radiation temperature equals the electron temperature when the electrons have a Maxwellian velocity distribution.

The absolute magnitude of the emitted noise power is determined with a known blackbody reference noise source which is gated on while the reference sample is taken. The radiometer output is zero when the reference noise power equals the unknown noise power. For reasons of measurement accuracy it is preferable to use the radiometer as a null detector which eliminates the effect of gain fluctuations and gives the result independent of the receiver characteristics. However, measurements are less time consuming when the receiver output is calibrated with the reference source and the noise power measured absolutely.

The electron radiation temperature is measured when the blackbody reference noise source is turned on continuously. The first sample is the sum of the noise power emitted by the plasma column and that part of the reference noise power which is not absorbed by the column. The second sample is given by the reference noise power alone. The sample difference becomes

$$
\begin{array}{r}
\Delta P=\left[A k T_{\mathrm{rad}} B+(1-A) k T_{\mathrm{ref}} B\right] \\
-\left[k T_{\mathrm{ref}} B\right]=A k\left(T_{\mathrm{rad}}-T_{\mathrm{ref}}\right) B .
\end{array}
$$

Thus, when the plasma column has a nonvanishing absorption coefficient the radiation temperature is found by adjusting the known reference temperature for zero radiometer output.

When a reference noise source with excess noise figure $F$ is used and the noise power varied with an attenuator at room temperature $\left(T_{0}=290 \mathrm{~K}\right)$ the reference temperature is given by

$$
T_{\text {ref }}=T_{0}\left[10^{(F-\alpha) / 10}+1-2 \times 10^{-\alpha / 10}\right],
$$

where $F$ and the attenuation $\alpha$ are given in decibels. The latter includes the insertion loss of the circulator between ports 1 and 2.

The finite isolation of the circulator can give rise to a measurement error due to interference between the small reverse component of the reference signal with the forward component which differs in samples $P_{1}$ and $P_{2}$. In the range of strong absorption the error is bounded by $\epsilon=2 \times 10^{-\gamma / 2}$ where $\gamma$ is the isolation in decibels. In the present case $\gamma=30 \mathrm{~dB}$ and $\epsilon=6.3 \%$, which is comparable to the temperature fluctuations. Note that, for given radiometer output fluctuations, the uncertainty in the temperature measurement increases as the absorption coefficient becomes smaller.

The power absorption coefficient is found by measuring the reflected power from the plasma column in the shorted waveguide. An incident microwave signal is gated on while the signal sample is taken. Choosing the incident power $P_{\text {ine }}$ about $30 \mathrm{~dB}$ above the noise emission level, the latter can be neglected and the radiometer output becomes proportional to

$$
\Delta P=(1-A) P_{\text {inc }} .
$$

Two calibration points are easily obtained. Complete absorption corresponds to zero radiometer output, while the zero absorption level is obtained when the plasma breakdown is turned off. The losses in the quartz tube and radiation from the holes in the waveguide sidewalls cause a negligibly small absorption.

Since the absorption coefficient is measured with a monochromatic signal source at a level well above noise level the measurements have a high frequency resolution and low fluctuations. Again the finite isolation of the circulator causes a measurement error

$$
\epsilon=2 \times 10^{-\gamma / 2} /(1-A)^{\frac{1}{2}} .
$$

It can be reduced by cascading two isolators or by canceling the reverse signal with a phase opposite signal provided by an external path between port 1 and 3 containing phase shifter and attenuator.

When the waveguide short is replaced by a matched termination, the same measurement technique yields the power reflection coefficient $\left(R^{\prime}\right)$ of the plasma column. The power transmission coefficient $\left(T^{\prime}\right)$ can be found by introducing the test signal at the side of the waveguide short. Complex measurements are made by means of a slotted line which is inserted between the plasma column and the circulator. The microwave signal of the movable probe is applied to the radiometer input. Measuring the complex reflection coefficient for different terminations gives the scattering parameters of the equivalent fourterminal microwave circuit for the discontinuity. For the purpose of determining the plasma parameters, however, temperature and emission or absorption measurements are the significant ones.

\section{EVALUATION OF MEASUREMENTS}

The absorption coefficient of extraordinary waves in a cold plasma shows a resonant behavior at the upper hybrid frequency $\omega=\left(\omega_{c}^{2}+\omega_{p}^{2}\right)^{\frac{1}{2}}$ where $\omega_{c}$ is the electron cyclotron frequency $\left(\omega_{c}=e B / m\right)$ and $\omega_{p}$ is the electron plasma frequency $\left(\omega_{p}^{2}=n e^{2} / m \epsilon_{0}\right) .{ }^{6}$ Due to the diffusion process the electron density at a given time in the afterglow falls from a maximum value in the column center to zero at the wall. In a uniform magnetic field this spread in density gives rise to a spectrum of upper hybrid

\footnotetext{
${ }^{6}$ A. F. Kuckes and A. Y. Wong, Phys. Fluids 8, 1161 (1965).
} 
FIG. 4. Power absorption times in the afterglow. The upper hybrid resonances lie in the range $\left(1-\omega^{2} \max / \omega^{2}\right)$ $\leq \omega_{c} / \omega \leq 1$. The narrow absorption line at $\omega_{c} / \omega \geq 1$ is a side effect due to the fringing fields of the holes in the waveguide sidewalls. coefficient vs normalized magnetic field at different

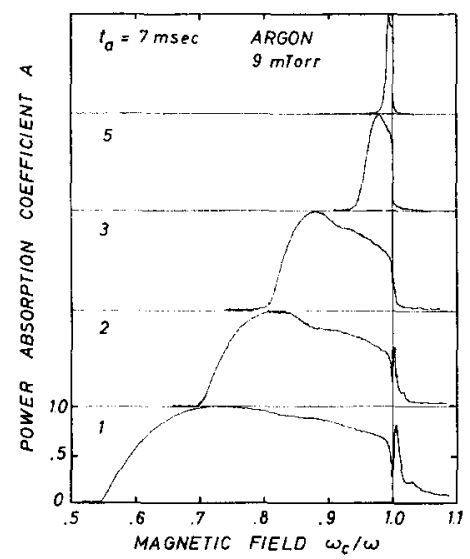

frequencies. Resonant absorption can be observed at a fixed frequency within the range of normalized magnetic fields $\left[1-\left(\omega_{p \max }{ }^{2} / \omega^{2}\right)\right]^{i} \leq \omega_{c} / \omega \leq 1$.

Typical absorption measurements for different afterglow times are shown in Fig. 4. From the lower onset of absorption $\left(\omega_{c} / \omega\right)_{0}=\left[1-\left(\omega_{p \max }^{2} / \omega^{2}\right)\right]^{\frac{1}{2}}$ one finds the maximum electron density. With increasing afterglow time the density decays and the onset point shifts toward $\omega_{c} / \omega=1$ where resonance occurs for vanishing density. Even in the late afterglow the plasma column behaves in the resonance range like a blackbody $(A=1)$ which permits an accurate measurement of low electron temperatures. The narrow absorption line for $\omega_{c} / \omega \gtrsim 1$ is a side effect due to the fringing fields of the holes in the waveguide side walls.

The density decay, as derived from the absorption onset, is shown in Fig. 5. The exponential decay results from ambipolar diffusion of electrons and ions to the walls. Electron densities in the range of $n_{e}=10^{9} \cdots 10^{11} \mathrm{~cm}^{-3}$ are conveniently measured. From the decay time one obtains collision data. Since by Kirchhoff's law the emission is

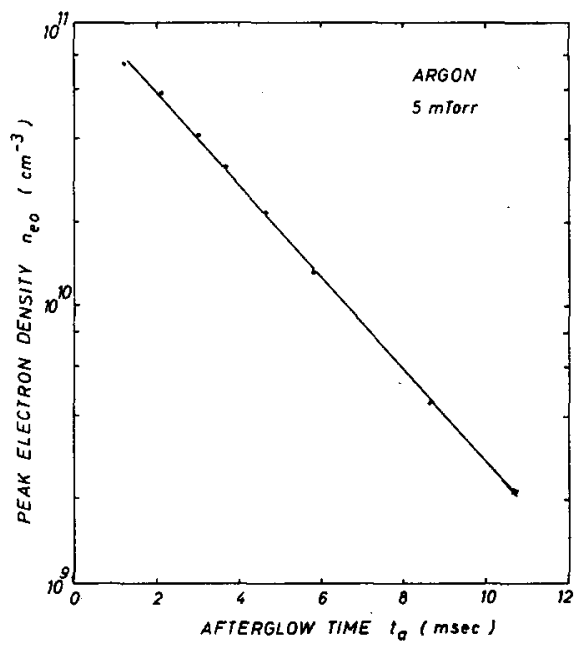

FIG. 5. Electron density decay in the center of the plasma column as derived from the onset of absorption.
FIG, 6. Electron temperature decay in the center of the plasma column.

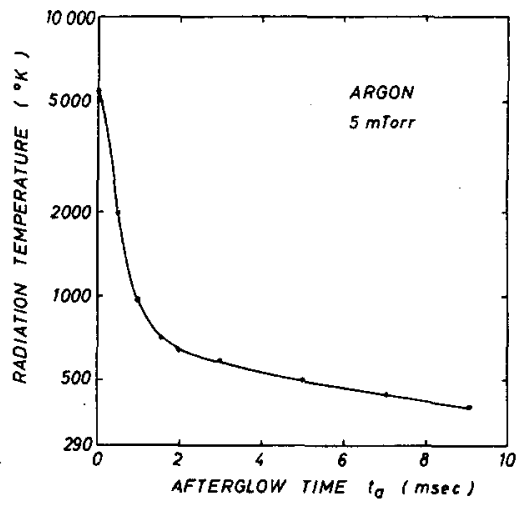

proportional to the absorption, one can also determine the electron density from the emission spectrum.

The radiation temperature vs afterglow time is found by the direct measurement described in Sec. IV. Under the present experimental conditions $\left(n_{e} \simeq 10^{11} \mathrm{~cm}^{-3}\right.$ and $T_{e} \simeq 6000 \mathrm{~K}$ at $t_{a}=0$ ) the electron velocity distribution relaxes to a Maxwellian within less than $1 \mu \mathrm{sec}$ so that for $t_{a} \gg 1 \mu \mathrm{sec}$ the measured radiation temperature equals the true electron temperature. Figure 6 shows the measured temperature decay of the electrons in the center region of the plasma column. The electrons lose their excess energy in elastic collisions with ions and neutrals. They can gain energy in collisions with metastable ions and thereby slow down the temperature decay as indicated in the later afterglow.

In a nonuniform plasma column resonant absorption and emission occur in a well defined resonant layer as a theoretical calculation of the problem shows. ${ }^{7}$ At the onset magnetic field the upper hybrid resonance lies in the center of the column. The electron temperature measured near $\left(\omega_{c} / \omega\right)_{0}$ describes the conditions at the column center. Likewise, near $\omega_{c} / \omega=1$ the electron temperature characterizes the electrons in a layer near the wall where the density is low. Thus measuring the electron tempera-

Fig. 7. Electron temperature vs normalized magnetic field at different times in the afterglow. The variation is due to the shift of the upper hybrid resonance from the center of the column to the wall and thus reflects the spatial temperature variation. The temperature can be measured only in the range of nonzero absorption, i.e., in the hybrid frequency range.

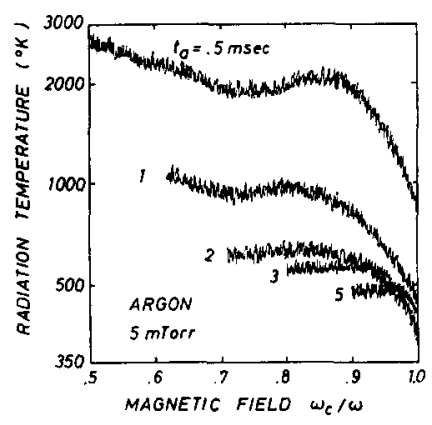

${ }^{7}$ R. W. Gould and R. H. Ault, Bull. Amer. Phys. Soc. 13, 888 (1968); R. H. Ault (private communication). 
ture vs magnetic field at a fixed afterglow time shows the radial temperature variation.

Figure 7 shows several measure temperature profiles at different times in the afterglow. In the center region of the plasma the electron temperature is essentially uniform but there is a pronounced temperature drop toward the side walls.

\section{ACKNOWLEDGMENTS}

The authors are indebted to Professor R. S. Harp for interesting discussions and suggestions during the course of this research. In addition, we benefitted from Professor A. Moffet's experience in designing a low noise i.f. preamplifier. This work was supported by the U. S. Atomic Energy Commission.

THE REVIEW OF SCIENTIFIC INSTRUMENTS

VOLUME 40, NUMBER 11

NOVEMBER 1969

\title{
A Light Pulse Demodulator with Large Bandwidth and High Sensitivity
}

\author{
Charles E. Tyier* \\ Arthur Holly Compton Laboratory of Physics, Washington University, Saint Louis, Missouri 63130
}

(Received 1 April 1969; and in final form, 22 July 1969)

\begin{abstract}
A pulse demodulation technique is described which, by employing the zero crossing technique, enables one to avoid the usual photomultiplier bandwidth limitation imposed by the anode risetime. The sensitivity limitation imposed by the photoelectric quantum noise is automatically eliminated. A light pulse with 0.4 nsec risetime is demodulated.
\end{abstract}

$\mathrm{P}^{\mathrm{R}}$ RIOR to the invention of the zero crossing discriminator, ${ }^{1-4}$ timing precision of photomultiplier anode pulses was limited to essentially the risetime of the pulses because of their characteristic amplitude fluctuations. ${ }^{5,6}$ That is, while a conventional amplitude discriminator will trigger on a large pulse early in its rise and on a small pulse late in its rise, a zero crossing discriminator triggers when a shorted stub of transmission line reflects an inverted and delayed pulse which pulls the anode signal back through zero, so that the timing of the pulse is essentially independent of its amplitude. The present work shows how the zero crossing technique can be used to increase photomultiplier bandwidth in optical pulse demodulation applications.

Consider a sequence of light pulses, each described by the photon density $I(\lambda, t)$, where $\lambda$ is the optical wavelength and $t$ gives the time behavior of the pulse envelope. If such a pulse is incident on a photocathode whose quantum efficiency is $q(\lambda)$, then the probability there is no primary photoelectron prior to the time $t$ is readily

\footnotetext{
* This investigation was supported in part by Public Health Service Fellowship No. 5 FO1 GM38076-02 from the National Institute of General Medical Sciences and in part by NASA Grant NSG581. The author is presently at the Hewlett-Packard Physical Electronics Laboratories, 1501 Page Mill Road, Palo Alto, Calif. 94304 .

1 P. Orman, Nucl. Instrum. Methods 21, 121 (1963).

2 D. L. Wieber and H. W. Lefevre, IEEE Trans. Nucl. Sci. NS-13, No. 1, 406 (1966).

${ }^{3}$ Instruction Manual No. B-3658, EG\&G, Inc., 35 Congress St., Salem, Mass. 01970.

${ }^{4}$ D. A. Gedcke and J. W. McDonald, Nucl. Instrum. Methods 58, 253 (1968).

${ }^{5}$ F. J. Lombard and F. Martin, Rev. Sci. Instrum. 32, 200 (1961).

${ }^{6}$ J. R. Prescott, Nucl. Instrum. Methods 39, 173 (1966).
}

calculated to be (on Poisson statistics for the photons)

$$
\begin{aligned}
P(0 \mid I(\lambda, t), q(\lambda), t) & =\exp \left[-\int_{0}^{t} \int_{0}^{\infty} q(\lambda) I\left(\lambda, t^{\prime}\right) d \lambda d t^{\prime}\right] \\
& =\exp \left[-Q \int_{0}^{t} \mathfrak{I}\left(t^{\prime}\right) d t^{\prime}\right]
\end{aligned}
$$

For a Kerr cell modulating a laser of wavelength $\lambda$, the photon density conveniently separates into a product of densities in time and in wavelength so that one has

$$
I(\lambda, t)=I^{\prime}(t) \delta\left(\lambda-\lambda_{0}\right) .
$$

Similarly, for a pulsed light emitting diode, one has

$$
I(\lambda, t)=I^{\prime}(t) I^{\prime \prime}(\lambda)
$$

The probability density function for the liberation of the first primary photoelectron after the pulse begins is

$$
\begin{aligned}
p(1 \mid I(\lambda, t), q(\lambda), t) & =-(\partial / \partial t) P(0 \mid I(\lambda, t), q(\lambda), t) \\
& =Q g(t) \exp \left[-\int_{0}^{t} Q \mathfrak{g}\left(t^{\prime}\right) d t^{\prime}\right] .
\end{aligned}
$$

Let the pulse begin at $t=0$ and end at $t=T$. Since $Q \mathcal{g}(t)$ is the liberation rate of primary photoelectrons, if the incident light be made feeble enough that the probability of liberation of a single photoelectron is small, say,

$$
(Q \mathfrak{g}(t))^{-1}>10 T,
$$

then

$$
p(1 \mid I(\lambda, t), q(\lambda), t)=Q \mathfrak{g}(t)[1-\mathcal{O}\{Q \mathfrak{g}(t) T\}] \approx Q \mathfrak{g}(t) .
$$

